\title{
Cast Shadow Removal with GMM for Surface Reflectance Component
}

\author{
Zhou Liu, Kaiqi Huang, Tieniu Tan, Liangsheng Wang \\ National Lab of Pattern Recognition, Institute of Automation, \\ Chinese Academy of Sciences, Beijing, China, 100080 \\ Email: \{zliu,kqhuang,tnt, lswang\}@nlpr.ia.ac.cn
}

\begin{abstract}
Cast shadow on the background is generated by an object moving between a light source and the background. The position and illumination of the source always change with time, while the background is stable. Therefore, features connected with light source always change with time, such as geometry and color. In this paper, we present a shadow removal method by homomorphic model to extract surface reflectance component, which is only connected with background of the scene and is robust to change of light source. We assume that reflectance component fits Gaussian distribution, and then use GMM to model it. Experimental results show that, except dealing with shadow, our method is not sensitive to the change of illumination
\end{abstract}

\section{Introduction}

Moving shadow detection is critical for object detection in visual surveillance system, since shadow always causes problems, such as object merging and shape distortion. It is also a challenge for object detection. First, shadow points are detectable as foreground, since they differ significantly from the background. Second, shadow has the same motion as the object casting it. Third, many features used to remove shadow, such as color, are not very robust.

Much has been done on the shadow detection. Cucchiara et.al [1] first defined an approach of shadow detection based on HSV color space. They assume that the $\mathrm{V}$ component is smaller than its priori value, when shadowed. It needs to tune up four parameters every time the context changes. The authors in [2] use YUV information to reduce time consumed by the transformation to HSV color space. Martel-Brisson et al. [3] assume that, for a given pixel, the shadow cast by different moving foreground objects is relatively similar and model every pixel by Gaussian Mixture
Shadow Model(GMSM). The comparative and evaluation study can be found in [4].

Cast shadow on the background is generated by an object moving between a light source and the background [5]. The position and illumination of the source always change with time, while the background is stable. Therefore, features connected with light source always change with time. However, currently, there are many approaches for moving shadow removal which are connected with light source, more or less, such as [1].

The surface reflectance component which is only connected with the surface of the scene is robust to the change of light source. In this paper, we present a shadow removal method by homomorphic filtering to extract surface reflectance component based on a shadow model [5]. In [6] and [7], the homomorphic filter is also used to remove shadow, but their background method can not deal with periodical motion, which is usual in outdoor scene. Moreover, we will explain why homomorphic filter can be used to remove shadow under certain condition from the view of shadow's birth.

Because the GMM (Gaussian Mixture Model) can deal with slow lighting changes, periodical motions, etc, there are many applications as [8]. We combine it with homomorphic model to remove shadow. In the model, we assume the surface reflectance value is Gaussian distribution. Experimental results show that our method is not sensitive to the change of illumination.

This paper is organized as follows: in Section 2, the principle of our algorithm is provided; its integration with GMM is shown in Section 3; in Section 4, the result is analyzed and conclusions follow it.

\section{Principle of our method 2.1. Cast shadow modeling}

According to the classification in [5], shadows are composed of two parts: self shadow and cast shadow. 
The cast shadow is the area projected on the scene by the object and can be further classified as umbra and penumbra. The umbra corresponds to the area where the direct light is totally blocked by the object, while the penumbra is partially blocked, as in Figure 1. In this paper, we deal with cast shadow removing.

The intensity of a pixel in image, at time instant $t$, can be modeled as [9]:

$$
s_{t}(x, y)=E_{t}(x, y) \rho_{t}(x, y)
$$

Where $x$ and $y$ are the coordinates of pixel, $E_{t}(x, y)$ is the irradiance and $\rho_{t}(x, y)$ is the reflectance of the object surface.

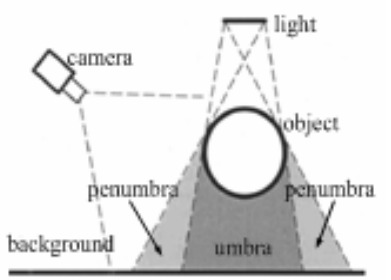

Figure 1. Generation of shadows: umbra is totally blocked and penumbra partially blocked [5].

As described in [5], $E_{t}(x, y)$ can be computed as follows:

$$
\begin{aligned}
& E_{t}(x, y) \\
& =\left\{\begin{array}{cc}
c_{A}+c_{P} \cos \angle(N(x, y), L), & \text { if illuminated } \\
c_{A}+k(x, y) c_{P} \cos \angle(N(x, y), L), & \text { if penumbra } \\
c_{A}, & \text { if umbra }
\end{array}\right.
\end{aligned}
$$

Where $c_{A}$ and $c_{P}$ are the intensity of the ambient light and of the light source, $L$ the direction of the light source, $N(x, y)$ the object surface normal. $k(x, y)$ describes the transition inside the penumbra and depends on the light source and scene geometry, obviously, $0 \leq k(x, y) \leq 1$.

\subsection{Homomorphic model for illumination invariant component}

From model (2), we find that the scene illumination component $E_{t}(x, y)$ changes slowly, except the transition from the illuminated area to umbra area (i.e. penumbra), whereas the reflectance component contains medium high-frequency details (i.e. object information) [9]. We make two assumptions: penumbra changes slower than reflectance value of the surface and the area of shadow is not too small. As described in [5], it is always true that penumbra changes slower than texture/object edge. At this case, after filtering, we always can find a threshold to partition the contour of shadow and texture/object edge, as shown later. However, it is not true when the illumination is strong and the contrast is low. And then, our method will fail.

In (1), we find that only the $\rho_{t}(x, y)$ component is connected with the object surface. Therefore, if we can separate $\rho_{t}(x, y)$ and $E_{t}(x, y)$ component, we can easily remove the shadow. Furthermore, without using color information, it can overcome some shortcomings of the shadow removal by color feature. As described above, the $\rho_{t}(x, y)$ component has more medium high-frequency component. Here, we use homomorphic filtering to extract the reflectance component [9]. The flow chart is described in Figure 2.

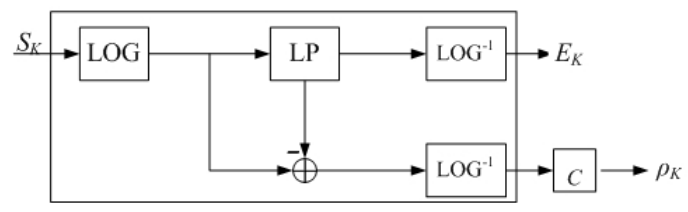

Figure 2. Flow chart of homomorhpic filtering.

In Figure 2, we can see that after logarithm (LOG), the image is lowpass filtered by a Gaussian kernel, then subtracted from the logarithmic original, and we get the reflectance component multiplied by a constant $C$ and see it as the last value of pixels. Of course, the illumination component also has some medium frequency component and the separation of $\rho_{t}(x, y)$ and $E_{t}(x, y)$ is only an approximation. This can be seen in Figure 3.

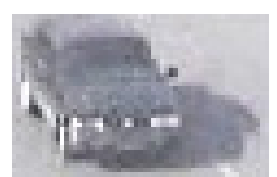

(a) Original image

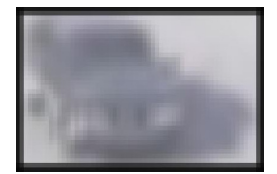

(b) Illumination component

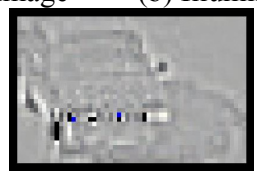

(c) Reflectance componen

Figure 3. Example of homomorphic filtering.

In Figure 3(b) and Figure 3(c) show the illumination and reflectance component respectively. In Figure 3(c), the contour of the shadow is still evident, but the inner part of shadow is same as the surface. Section 4 will show that, after subtraction by the background and thresholding, the contour can be removed. 


\section{GMM for reflectance component}

The GMM can deal with slow illumination changes, periodical motions from clutter background, etc. However, it can not deal with shadows and fast illumination changes [8]. In this paper, we incorporate this background modeling method with our shadow removing method.

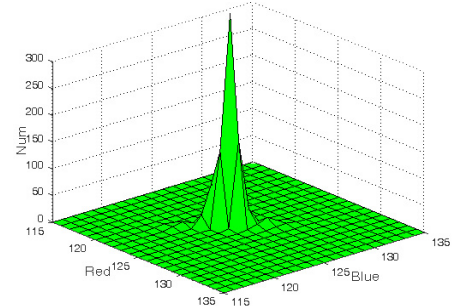

Figure 4. Histogram of red and blue reflectance components of a single pixel's distribution in a video. It fits Gaussian distribution very well.

In [10], Stauffer and Grimson model the RGB value history by a mixture of $K$ Gaussian distributions, while, in our paper, we use the $K$ Gaussian distributions to model the history of surface reflectance value. After homomorphic filtering, values of each pixel represent a measurement of the reflectance of object surface for RGB spectra. We consider the reflectance component values of a particular pixel over time as a "pixel process". As shown in Figure 4, the reflectance components fit Gaussian distribution well. With a static background and without the requirement of static lighting, that value would be relatively constant. For a pixel, the probability of reflectance value, at time $t$, can be modeled as:

$$
P\left(X_{t}\right)=\sum_{i=1}^{K} \omega_{i, t} * \eta\left(X_{t}, \mu_{i, t}, \sum_{i, t}\right)
$$

where $K$ is the number of distributions and 3 is used in this paper, $\omega_{i, t}$ is an estimate of the weight of the $i^{t h}$ Gaussian, $\mu$ is the mean, $\sum_{i, t}$ is the covariance matrix of the $i^{\text {th }}$ Gaussian, and $\eta$ is a Gaussian probability density function

$$
\eta\left(X_{t}, \mu_{i, t}, \sum_{i, t}\right)=\frac{1}{(2 \pi)^{n / 2}|\Sigma|^{1 / 2}} e^{-\frac{1}{2}\left(X_{t}-\mu_{t}\right)^{T} \sum^{-1}\left(X_{t}-\mu_{t}\right)}
$$
form

We also assume that the covariance matrix is of the

$$
\Sigma_{k}=\sigma_{k}^{2} I
$$

This means that the red, green and blue reflectance components of the surface are independent and have the same variances, which can reduce costly matrix computation. The weight $\omega_{i, t}$ is adjusted as follows:

$$
\omega_{t}=(1-\alpha) \omega_{t-1}+\alpha\left(M_{t}\right)
$$

where $\alpha$ is the learning rate and $M_{t}$ is 1 for the model which is matched and 0 for others. After ordering the Gaussians, the first B distributions are chosen as the background model:

$$
\left.B=\arg \min _{b}\left(\sum_{k=1}^{b} \omega_{k}\right\rangle T\right)
$$

Where, $T$ is a measure for minimum models for background. More details may be found in [10]. Now, we can see that, in our method, after determination of the Gaussian kernel and constant $C$, the remaining parameters are only in the GMM model.

However, for objects with little high-frequency information, hollows will appear. At this case, as long as the assumption can be satisfied, we can still get the contour and then fill the hollows. Some other rules can also be used to improve quality. For example, if a pixel is a shadow, then it must satisfy $0 \leq I_{k}^{V}(x, y) / B_{k}^{V}(x, y) \leq 1$ [1]. However, at this time, another background in HSV color space should be constructed. The result after post-processing is shown as in Figure 5.

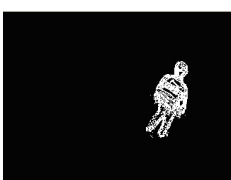

(a) original image

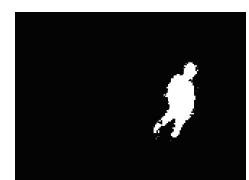

(b) result of post-processing Figure 5. Result after post-processing

\section{Experiments and results}

In this section, four videos with size of $320 \times 240$ are used to demonstrate the performance of our method. In this part, we will see that our method is not sensitive to the change of illumination. HighwayI_raw and intelligentroom_raw videos are downloaded from http://cvrr.ucsd.edn/aton/shadow. HighwayI_raw video as Figure 6(a) is also used in [1], where the shadow is very dark and its illumination is low. Usualway_raw video as Figure 6(b) is recorded outside our lab, where the color of the shadow is medium and the illumination is strong. Intelligentroom_raw video as Figure 6(c) is indoor environment and has the lightest shadow and the shadow is highlighted in red. Figure 7 shows the video under fast illumination.

Figure 6 shows result of our method to these three videos. Identical parameters are used in three videos. 
The Gaussian lowpass kernel is $5 \times 5$ with variance 13 . Other parameters are: $C=125, \alpha=0.02, T=0.6$, $\sigma_{k}^{2}=6$. We can see that, despite change of environment, our method still works well. Our method appears to be insensitive to the change of illumination and environment. Figure 6(b) shows that the contour of shadow can be removed at the expense of object integrity by selecting parameters.

Figure 7 shows the result of our method under fast illumination environment. It shows that our method does well under this condition.
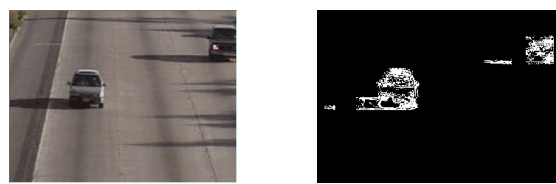

(a) highwayI_raw

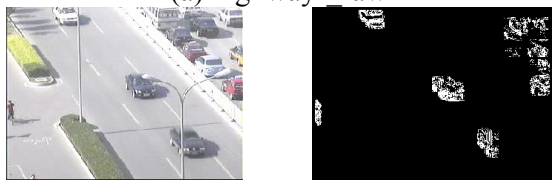

(b) usualway raw

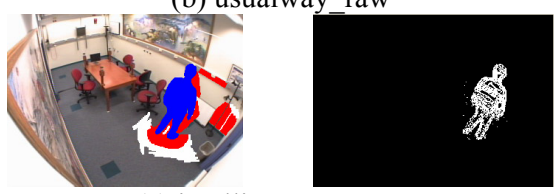

(c) intelligentroom_raw

Figure. 6. Performance of our method. It changes little under different environment and illumination.

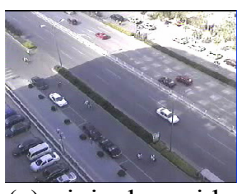

(a)original video

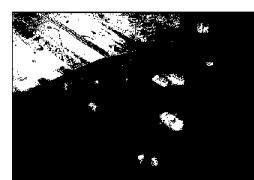

(b)object detection GMM(RGB value)

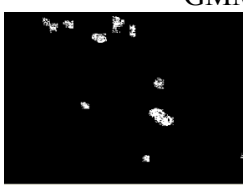

(c)object detection after homomorphic filtering

Figure. 7. Performance under fast illumination change

\section{Conclusions and future work}

It is a challenge to remove shadows, since many features used to remove shadow, such as color, are not very robust. In this paper, we extract surface reflectance component to remove the shadow according to the model described in [5]. Then, we model the reflectance component by GMM. Experimental results show that our method is not so sensitive to the change of illumination. However, our method is based on the assumption that the penumbra changes slower than texture/object edges, so it may fail under strong shadow. Also, when object has little highfrequency information, there are hollows. Our future work will focus on how to cooperate our method with other features, such as color and geometry to get a better performance, and modified filter to get reflectance component more accurately.

\section{Acknowledgment}

This work is supported by research grants from the National Basic Research Program of China (No. 2004CB318110), the National Natural Science Foundation of China (No. 60121302, 60335010) and the International Cooperation Program of Ministry of Science and Technology of China (Grant No. 2004DFA06900).

\section{References}

[1] R.Cucchiara, C.Grana, M. Piccardi et al., "Improving Shadow Suppression in Moving Object Detection with HSV Color Information" Proc. IEEE Int'l Conf. Intelligent Transportation Systems, pp.334-339, Aug. 2001.

[2] O.Schreer, I. Feldmann, U. Golz et al., "Fast and Robust Shadow Detection in Videoconference Applications", 4th IEEE Intern. Symposium on Video Proces. and Multimedia Comm., pp. 371-375, 2002.

[3] Martel-Brisson, and André Zaccarin, "Moving Cast Shadow Detection from a Gaussian Mixture Shadow Model", Proc. IEEE Int'l Conf. Computer Vision and Pattern Recognition, Vol. 2, pp. 643-648, June 2005.

[4] A. Prati, I. Mikic , M.M. Trivedi, and R. Cucchiara, "Detecting Moving Shadows: Algorithms and Evaluation", IEEE Trans. PAMI, vol. 25, pp. 918-923, 2003

[5] J. Stauder, R. Mech, J. Ostermann, "Detection of Moving Cast Shadows for Object Segmentation", IEEE Transactions on Multimedia, vol.1, no. 1, Mar. 1999, pp. 65-76.

[6] S. Y. Chien, S. Y. Ma, and L. G. Chen, "Efficient moving object segmentation algorithm using background registration technique", IEEE Trans. Circuits Syst. Video Technol., vol. 12, no. 7, pp. 577-586, Jul. 2002.

[7] Jianguang Lou, Hao Yang, Weiming Hu and Tieniu Tan, "An Illumination Invariant Change Detection Algorithm", Proceedings of 5th Asian Conference on Computer Vision (ACCV), 2002.

[8] Ying-Li Tian, M. Lu, A. Hampapur, "Robust and efficient foreground analysis for real-time video surveillance", Proc. IEEE Int'l Conf. Computer Vision and Pattern Recognition, 2005, pp. 1182 - 1187.

[9] A.V.Oppenheim, R. Schafer, T. Stockham, "Nonlinear filtering of multiplied and convolved signals", Proceedings of the IEEE, 56(8), 1968, pp: 1264-1291.

[10] C.Stauffer and W.E.L. Grimson, "Adaptive Background Mixture Models for Real-Time Tracking," Proc. IEEE Int'l Conf. Computer Vision and Pattern Recognition, 1999, pp. 246-252. 